\title{
Serendipitous discovery of a dusty disc around WDJ181417.84-735459.83
}

\author{
E. González Egea ${ }^{\oplus},{ }^{1 \star}$ R. Raddi ${ }^{\circledR},{ }^{2 \star}$ D. Koester, ${ }^{3}$ L. K. Rogers ${ }^{\circledR},{ }^{4}$ F. Marocco ${ }^{\oplus}, 5,6$ W. J. Cooper ${ }^{\circledR},{ }^{1,7}$ \\ J. C. Beamin ${ }^{\oplus},{ }^{8}$ B. Burningham ${ }^{\oplus},{ }^{1}$ A. Day-Jones, ${ }^{9}$ J. Forbrich ${ }^{\oplus 1}$ and D. J. Pinfield ${ }^{\oplus 1}$ \\ ${ }^{1}$ Centre for Astrophysics Research, University of Hertfordshire, Hatfield AL10 9AB, UK \\ ${ }^{2}$ Departament de Física, Universitat Politècnica de Catalunya, c/Esteve Terrades 5, Castelldefels E-08860, Spain \\ ${ }^{3}$ Institut für Theoretische Physik und Astrophysik, Christian-Albrechts-Universität, Kiel 24118, Germany \\ ${ }^{4}$ Institute of Astronomy, University of Cambridge, Madingley Road, Cambridge CB3 OHA, UK \\ ${ }^{5}$ IPAC, Mail Code 100-22, California Institute of Technology, 1200 E. California Blvd., Pasadena, CA 91125, USA \\ ${ }^{6}$ Jet Propulsion Laboratory, California Institute of Technology, 4800 Oak Grove Drive, M/S 169-327, Pasadena, CA 91109, USA \\ ${ }^{7}$ Istituto Nazionale di Astrofisica, Osservatorio Astrofisico di Torino, Strada Osservatorio 20, I-10025 Pino Torinese 24118, Italy \\ ${ }^{8}$ Núcleo de Astroquímica y Astrofísica, Instituto de Ciencias Químicas Aplicadas, Facultad de Ingeniería, Universidad Autónoma de Chile, Av. Pedro de \\ Valdivia 425, Santiago 7500912, Chile \\ ${ }^{9}$ Earl Mortimer College, South Street, Leominster, Herefordshire HR6 8JJ, UK
}

Accepted 2020 December 9. Received 2020 December 8; in original form 2020 October 15

\begin{abstract}
Spectroscopic observations of white dwarfs reveal that many of them are polluted by exoplanetary material, whose bulk composition can be uniquely probed this way. We present a spectroscopic and photometric analysis of the DA white dwarf WDJ181417.84-735459.83, an object originally identified to have a strong infrared (IR) excess in the 2MASS and WISE catalogues that we confirmed to be intrinsic to the white dwarf, and likely corresponding to the emission of a dusty disc around the star. The finding of $\mathrm{Ca}, \mathrm{Fe}$, and $\mathrm{Mg}$ absorption lines in two X-SHOOTER spectra of the white dwarf, taken 8 years apart, is further evidence of accretion from a dusty disc. We do not report variability in the absorption lines between these two spectra. Fitting a blackbody model to the IR excess gives a temperature of $910 \pm 50 \mathrm{~K}$. We have estimated a total accretion flux from the spectroscopic metal lines of $|\dot{\mathrm{M}}|=1.784 \times 10^{9} \mathrm{~g} \mathrm{~s}^{-1}$.
\end{abstract}

Key words: accretion, accretion discs - stars: white dwarfs.

\section{INTRODUCTION}

White dwarfs, the final evolutionary stage of low-to-intermediate mass stars, are compact objects whose photospheres typically show absorption spectral lines of hydrogen, helium, or do not show any lines (DA, DB, or DC white dwarf spectral type, respectively). Due to their high surface gravities, with $\log g \sim 8\left[\mathrm{~cm} \mathrm{~s}^{-2}\right]$, elements heavier than $\mathrm{H}$ and $\mathrm{He}$ rapidly diffuse from the convective envelope into the interior, with time-scales much shorter than the white dwarfs' cooling time (e.g. Fontaine \& Michaud 1979; Paquette et al. 1986; Koester 2009).

However, traces of metals are found in white dwarfs' spectra as absorption lines, in which case the white dwarfs are classified as DAZ, DBZ, or DZ. These metals were initially thought to come from accretion of the interstellar medium (e.g. Bruhweiler \& Kondo 1981; Aannestad \& Sion 1985; Dupuis et al. 1993), but were later alternatively explained by ongoing accretion from exoplanetary material around the white dwarf (Farihi et al. 2010). To date, we know of several hundreds of white dwarfs with atmospheric metals, representing 25-50 per cent of the spectroscopically confirmed white dwarfs (e.g. Zuckerman et al. 2003; Koester et al. 2005; Zuckerman et al. 2010; Hollands et al. 2017). Dusty discs have been observed as

^E-mail: e.gonzalez-egea@herts.ac.uk (EGE); roberto.raddi@upc.edu (RR) an excess in the infrared (IR) emission of the white dwarf in dozens of these cases, in around 1-4 per cent of white dwarfs (e.g. Farihi, Jura \& Zuckerman 2009; Debes et al. 2011; Barber et al. 2014; Rocchetto et al. 2015). In a few systems, Ca II triplet and other metallic emission lines are also detected in the white dwarf's spectrum, associated with a gaseous component of the accretion disc (e.g. Gänsicke et al. 2006; Manser et al. 2020).

The current widely accepted explanation for the formation of circumstellar discs around white dwarfs is the tidal disruption of asteroids (Jura 2003). In this model, either a large asteroid or several small ones (Wyatt et al. 2014) are tidally disrupted when entering the Roche radius of the white dwarf, forming an opaque flat ring or rings of dust with an extension of less than one solar radius, in a Saturn-like ring system. This flat ring model predicts the flux from the disc integrating the Planck function along the ring radius, with a radius-temperature dependence. The model successfully accounts for observations out to 24 microns taken by Spitzer space telescope (Fazio et al. 2004; Hora et al. 2004), with the caveat that 8 micron photometry can be contaminated in some cases by a silicate emission feature. This scenario includes also the possibility of planetesimals or any other exoplanetary material to be the parent bodies of these discs.

Earth-sized white dwarfs are intrinsically faint, and their observed discs are compact. It may be that many stars host discs that lie below the detection threshold (Rocchetto et al. 2015). Furthermore, IR excess may also be explained by a substellar companion in the 
system (e.g. Probst 1983), so a spectrum of the white dwarf is usually needed for the discrimination between the two scenarios, as discs have been found only around metal-polluted white dwarfs.

Variability in the IR, first detected by Xu \& Jura (2014) and suspected to be present in the majority of white dwarfs with discs (Swan, Farihi \& Wilson 2019a; Swan et al. 2020) is evidence of dynamical dust evolution, with no generally accepted explanation yet. Rogers et al. (2020) do not find variability in a near-IR monitoring campaign of a sample of white dwarfs with IR excesses, one interpretation being that tidal disruption events are rare and occur on short time-scales. There have been reported changes in spectral lines associated to circumstellar gas (e.g. Manser et al. 2016a, b; Dennihy et al. 2018). No definitive evidence for variability of absorption lines of metal pollutants has been found (von Hippel et al. 2007; Debes \& López-Morales 2008).

The analysis of the metal lines and discs found in white dwarfs can give us unique information about compositions of rocky planets (e.g. Jura \& Young 2014), asteroids, comets, and even gaseous giants (see recent discovery by Gänsicke et al. 2019). The dust composition is in some cases consistent with carbon-deficient and rocky material, likely similar to the material of the inner Solar System (e.g. Jura, Farihi \& Zuckerman 2009). We can infer the rate of the accretion of dusty material and abundances of metal species with respect to $\mathrm{H}$ or He abundances in white dwarfs' photospheres, while the accretion rates are consistent with parent bodies with sizes of the order of kilometers (Farihi 2016 and references therein). The most prevalent elements found in white dwarfs to date are oxygen, magnesium, aluminium, silicon, calcium, and iron (e.g. Gänsicke et al. 2012; Visscher \& Fegley 2013; Xu et al. 2019), elements that dominate also in the rocky bodies of the Solar System.

Here we report the discovery of photometric IR excess and absorption lines due to accretion of metals in a DAZ white dwarf, identified as WDJ181417.84-735459.83 by Gentile Fusillo et al. (2020b) (hereafter, WDJ1814-7354). In Section 2, we report the discovery of this object and present photometry and astrometry from the literature. In Section 3, we present follow-up observations: two epochs of X-SHOOTER spectroscopy in Section 3.1 and Spitzer photometry in Section 3.2. In Section 4, we discuss the analysis of the data: a white dwarf model fit to the spectroscopy in Section 4.1, determination of the composition of the accreted material and the diffusion time-scales in Section 4.2 and a disc model fit to the IR excess in Section 4.3. We discuss our results in Section 5. The conclusions are presented in Section 6.

\section{DISCOVERY OF WDJ1814-7354}

WDJ1814-7354 was originally identified by one of us (AD) as part of a search for unresolved white dwarf + ultracool dwarf benchmark binaries. The original sample of white dwarf candidates came from a selection of 36876 objects in the SuperCOSMOS Sky Survey (Hambly et al. 2001), following the same selection criteria as in Day-Jones et al. (2008). This list was then cross-matched with the All-Sky WISE catalogue (Cutri et al. 2013) to select targets with IR excess, that could potentially have an ultracool dwarf companion, obtaining 16928 objects. WDJ1814-7354 is one such outlier in the WISE (Wide Field Infrared Explorer, Wright et al. 2010) versus SuperCOSMOS colour-colour diagram presented in Fig. 1, lying $2.2 \sigma$ away from the median of the distribution of white dwarfs. The Gaia Data Release 2 (DR2; G87, G88) astrometry, photometry, and parameters of WDJ1814-7354 are presented in Table 1. The publicly available photometry for WDJ1814-7354 is summarized in Table 2.

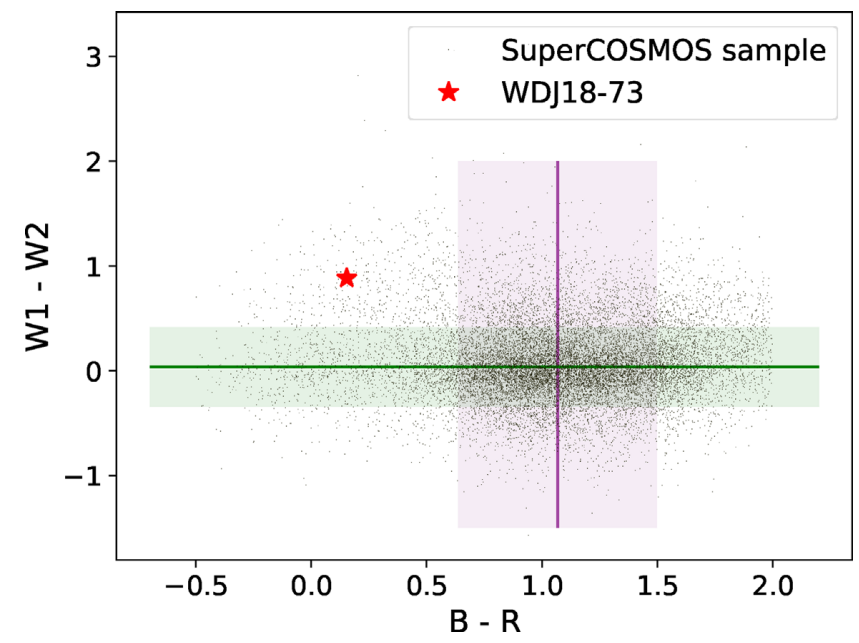

Figure 1. Colour-colour diagram of the sample of 16928 white dwarf candidates from Day-Jones et al. (2008) (shown as blue dots) originally selected from SuperCOSMOS that were also present in the WISE catalogue. The Y-axis shows the colour in WISE bands $\mathrm{W} 1$ and $\mathrm{W} 2$, and the $\mathrm{X}$-axis the colour in SuperCOSMOS B and R2 bands. The red star indicates the position on this diagram of WDJ1814-7354. The purple vertical line shows the position of the distribution's median in the Y-axis, and the $1 \sigma$ region is shown as the light purple shaded region. The green horizontal line and region shows the same for the $\mathrm{X}$-axis.

Table 1. Gaia DR2 astrometry and photometry of the object WDJ1814-7354, along with its $T_{\text {eff }}, \log g$ and distance (d) estimated in Section 4.1, and its distance (d) in parsecs from BailerJones et al. (2018). Barycentric right ascension $(\alpha)$ and declination $(\delta)$ are in the International Celestial Reference System and at Gaia DR2 reference epoch, 2015.5. Proper motion in right ascension $\left(\mu_{\alpha} \cos \delta\right)$ and declination $\left(\mu_{\delta}\right)$ and parallax $(\varpi)$ are also at Gaia DR2 reference epoch, 2015.5. Apparent magnitudes are in the three Gaia DR2 passbands.

\begin{tabular}{lc}
\hline Gaia source ID & Gaia DR2 6417955993895552128 \\
\hline$\alpha[\mathrm{deg}]$ & 273.57335015736 \\
$\delta[\mathrm{deg}]$ & -73.91738768154 \\
$\mu_{\alpha} \cos \delta\left[\mathrm{mas} \mathrm{yr}^{-1}\right]$ & $-63.89 \pm 0.08$ \\
$\mu_{\delta}\left[\mathrm{mas} \mathrm{yr}{ }^{-1}\right]$ & $-178.2 \pm 0.1$ \\
$\varpi[\mathrm{mas}]$ & $15.51 \pm 0.07$ \\
$\mathrm{G}[\mathrm{mag}]$ & $16.2028 \pm 0.0009$ \\
$\mathrm{G}_{\mathrm{BP}}[\mathrm{mag}]$ & $16.233 \pm 0.005$ \\
$\mathrm{G}_{\mathrm{RP}}[\mathrm{mag}]$ & $16.104 \pm 0.004$ \\
$\mathrm{G}_{\mathrm{BP}}-\mathrm{G}_{\mathrm{RP}}[\mathrm{mag}]$ & $0.129 \pm 0.009$ \\
$\mathrm{~d}[\mathrm{pc}]$ & $64.6 \pm 0.6$ \\
$T_{\text {eff }}[\mathrm{K}]$ & $10190 \pm 50$ \\
$\log g\left[\mathrm{~cm} \mathrm{~s}^{-1}\right]$ & $8.00 \pm 0.10$ \\
\hline
\end{tabular}

After comparing images and photometry from the Two Micron All Sky Survey (2MASS; Skrutskie et al. 2006) and VHS (VISTA Hemisphere Survey; McMahon et al. 2019) and from unWISE and WISE (see Fig. 2), we noted that the 2MASS and WISE detections are the blend of multiple objects. The WISE single source appears as two resolved sources in the unWISE catalogue, based on deeper imaging obtained after the coaddition of all 3-5 $\mu \mathrm{m}$ WISE images and with improved modeling of crowded regions (Schlafly et al. 2019). Three sources are detected by VHS.

Two objects are identified in Gaia DR2, one corresponding to the white dwarf and the other is identified as Gaia DR2 6417955993895551872, for which its Gaia DR2 information is 
Table 2. Apparent magnitudes $m$ of WDJ1814-7354 in different bands with central wavelengths $\lambda_{c}$ in micrometers and the full width at half maximum (FWHM) of the Point Spread Function (PSF) in arcseconds. S-COSMOS is an abbreviation of SuperCOSMOS.

\begin{tabular}{lcccc}
\hline Band (survey) & $\lambda_{c}[\mu \mathrm{m}]$ & $m[\mathrm{mag}]$ & Epoch & PSF ["] \\
\hline B (APASS9) & 0.444 & $16.49 \pm 0.0 .11$ & 2013.75 & 15 \\
B (S-COSMOS) & 0.467 & 16.345 & 1987.13 & - \\
g (APASS9) & 0.482 & $16.23 \pm 0.06$ & 2013.75 & 15 \\
GBP (GaiaDR2) & 0.513 & $16.233 \pm 0.005$ & 2015.5 & 0.1 \\
V (APASS9) & 0.554 & $16.113 \pm 0.011$ & 2013.75 & 15 \\
R2 (S-COSMOS) & 0.595 & 16.191 & 1987.13 & - \\
r (APASS9) & 0.625 & $16.19 \pm 0.06$ & 2013.75 & 15 \\
G (GaiaDR2) & $0.640^{a}$ & $16.2028 \pm 0.0009$ & 2015.5 & 0.1 \\
GRP (GaiaDR2) & 0.778 & $16.104 \pm 0.004$ & 2015.5 & 0.1 \\
I (S-COSMOS) & 0.807 & 16.115 & 1987.13 & - \\
J (2MASS) & 1.24 & $15.9 \pm 0.1$ & 2001 & 2.5 \\
J (VHS) & 1.252 & $16.078 \pm 0.009$ & 2019 & 0.51 \\
H (2MASS) & 1.65 & $15.49 \pm 0.13$ & 2013 & 2.5 \\
Ks (VHS) & 2.147 & $15.70 \pm 0.03$ & 2019 & 0.51 \\
Ks (2MASS) & 2.16 & $15.10 \pm 0.13$ & 2013 & 2.5 \\
W1 (AllWISE) & 3.35 & $13.94 \pm 0.03$ & 2010.59 & 6.1 \\
W1 (unWISE) & 3.35 & $14.290 \pm 0.03$ & - & 6.1 \\
W2 (AllWISE) & 4.60 & $13.07 \pm 0.03$ & 2010.59 & 6.4 \\
W2 (unWISE) & 4.60 & $13.433 \pm 0.03$ & - & 6.4 \\
W3 (AllWISE) & 11.6 & $10.76 \pm 0.09$ & 2010.31 & 6.5 \\
W4 (AllWISE) & 22.1 & $8.7 \pm 0.4$ & 2010.31 & 12 \\
\hline
\end{tabular}

Notes. ${ }^{a}$ Central wavelengths of Gaia passbands are from Weiler (2018).

${ }^{b}$ unWISE magnitudes were obtained by applying the relations in Finkbeiner et al. (2004) to the fluxes found in the unWISE catalogue: $1920 \pm 10$ nanomaggies for $\mathrm{W} 1$ and $4230 \pm 30$ nanomaggies for W2. Systematic uncertainties of $0.02 \mathrm{mag}$ were added (See Schlafly, Meisner \& Green 2019).

presented in Table 3. VHS and unWISE photometry for this source and the third source, identified as VHS 472908521370, is presented in Table 4. VHS 472908521370 has no counterpart in Gaia DR2. The angular separation from WDJ1814-7354 is $\sim 3.2$ arcseconds for VHS472908521370 and $\sim 6.3$ arcseconds for Gaia DR2 6417955993895551872. These additional objects are likely contributing to the 2MASS and WISE IR excess, but their contribution to optical photometry is negligible: Gaia DR2 6417955993895551872 is 3.5 mag fainter in the G-band with respect to WD1814-7354. VHS 472908521370 is even fainter, given its non-detection in Gaia DR2, which combined with its VHS colours indicates a very red object.

Gaia DR2 6417955993895551872 is likely a distant background object, as its proper motion is very small and not compatible with that of WDJ1814-7354, and its distance is not well constrained. This object has also photometric information in VHS catalogue but not in the unWISE catalogue.

\section{FOLLOW-UP OBSERVATIONS}

\subsection{X-SHOOTER spectroscopy}

\subsubsection{Observations in 2011}

WDJ1814-7354 was observed on 2011 September 18 with the multi-wavelength X-SHOOTER spectrograph (Vernet et al. 2011), mounted at the Cassegrain focus of the Very Large Telescope (VLT) UT2 in Cerro Paranal (Chile), as part of the ESO programme ID 087.C-0639B. The observing mode was SLITSPEC 100k/1pt/hg, longslit spectroscopy with auto-nodding along the slit. The three $\mathrm{X}$ SHOOTER arms covering the ultraviolet (UVB, 3000-5595 A), vis- ible (VIS, 5595-10240 Å), and near-infrared (NIR, 10 240-24 800 $\AA$ ) parts of the spectrum were used to take four subsequent exposures of 500 seconds each (590 in the case of the NIR arm), giving 2000 seconds of total exposure time for the UVB and VIS arms and 2360 seconds for the NIR arm. We used narrow slits of 1.0" width for the UVB arm and 0.9" for the VIS and NIR arms, giving spectral resolutions $R=\lambda / \Delta \lambda$ of 5400 (UVB), 8900 (VIS), and 5600 (NIR). The signal-to-noise ratio (SNR) achieved was around 40 for the VIS and 20 for the UVB part of the spectrum, enough for precise model fitting. The NIR part did not contain useful information for white dwarf modelling or abundance analysis. The weather conditions of the observing night were good, with seeing around 0.5-0.6", and the airmass was $1.5-1.6$ during the observations of this target.

\subsubsection{Observations in 2019}

WDJ1814-7354 was observed again in 2019 with X-SHOOTER as part of the ESO programme ID 0103.C-0431(B), with PI S. Xu. Stare mode was used, and the exposure times are as follows: 2 exposures of 1700 seconds with the UVB arm (a total of 3400 seconds) and 2 exposures of 1729 seconds with the VIS arm (3458 seconds total). The NIR part was not needed, so just one exposure of 100 seconds was taken. The SNR achieved was around 35 for the VIS and 20 for the UVB part of the spectrum. The weather conditions of the observing night were excellent, with seeing around $0.4-0.5$ ", and the airmass was around 1.6-1.7 during the observations.

To reduce both spectra, we used the ESO X-SHOOTER reduction pipeline (Modigliani et al. 2010) and the software ESOREFLEX. ${ }^{1}$

\subsection{Spitzer observations}

WDJ1814-7354 was observed with Spitzer on 2019 July 10 as part of the DDT program ID 14220, with PI Dr. Siyi Xu (see Xu et al. 2020), with the instrument and mode IRAC/Map PC, in both bands IRAC [3.6] and [4.5], centred at wavelengths 3.6 and 4.5 microns, respectively. The observing strategy was 30 seconds frame time with 11 medium-sized dithers for each wavelength.

The Spitzer [3.6] band image of WDJ1814-7354 is shown in Fig. 2 (bottom-left panel). Three sources are clearly distinguished in the Spitzer images. The relative fluxes of two of these sources (at the positions of WDJ1814-7354 and VHS 472908521370) were extracted after performing aperture photometry on successfully PSFfitted source positions using the stack of images, following Recipe 7 of the IRAC Instrument Handbook ${ }^{2}$ adapted to the commandline MOPEX (Makovoz \& Marleau 2005). Passive deblending of the sources was automatically performed with MOPEX routines, to take contamination from nearby sources into account. The uncertainty in Spitzer fluxes was the sum between the uncertainty obtained after the MOPEX extraction and the median values of the rms variations due to the relative repeatability of IRAC reported by Reach et al. (2005) of 1.7 per cent for channel 1 and 2.2 per cent in channel 2 .

The Spitzer fluxes were later corrected following section 7.9 of the IRAC Instrument Handbook. The first correction was applied by dividing the PRF fluxes by the correction factors in table C. 1 of the IRAC Instrument Handbook, whose values are 1.021 for IRAC1 and 1.012 for IRAC2. The second correction mentioned was not applied,

\footnotetext{
${ }^{1}$ Available at https://www.eso.org/sci/software/esoreflex/

${ }^{2}$ Available at https://irsa.ipac.caltech.edu/data/SPITZER/docs/irac/iracinstr umenthandbook/IRAC_Instrument_Handbook.pdf
} 


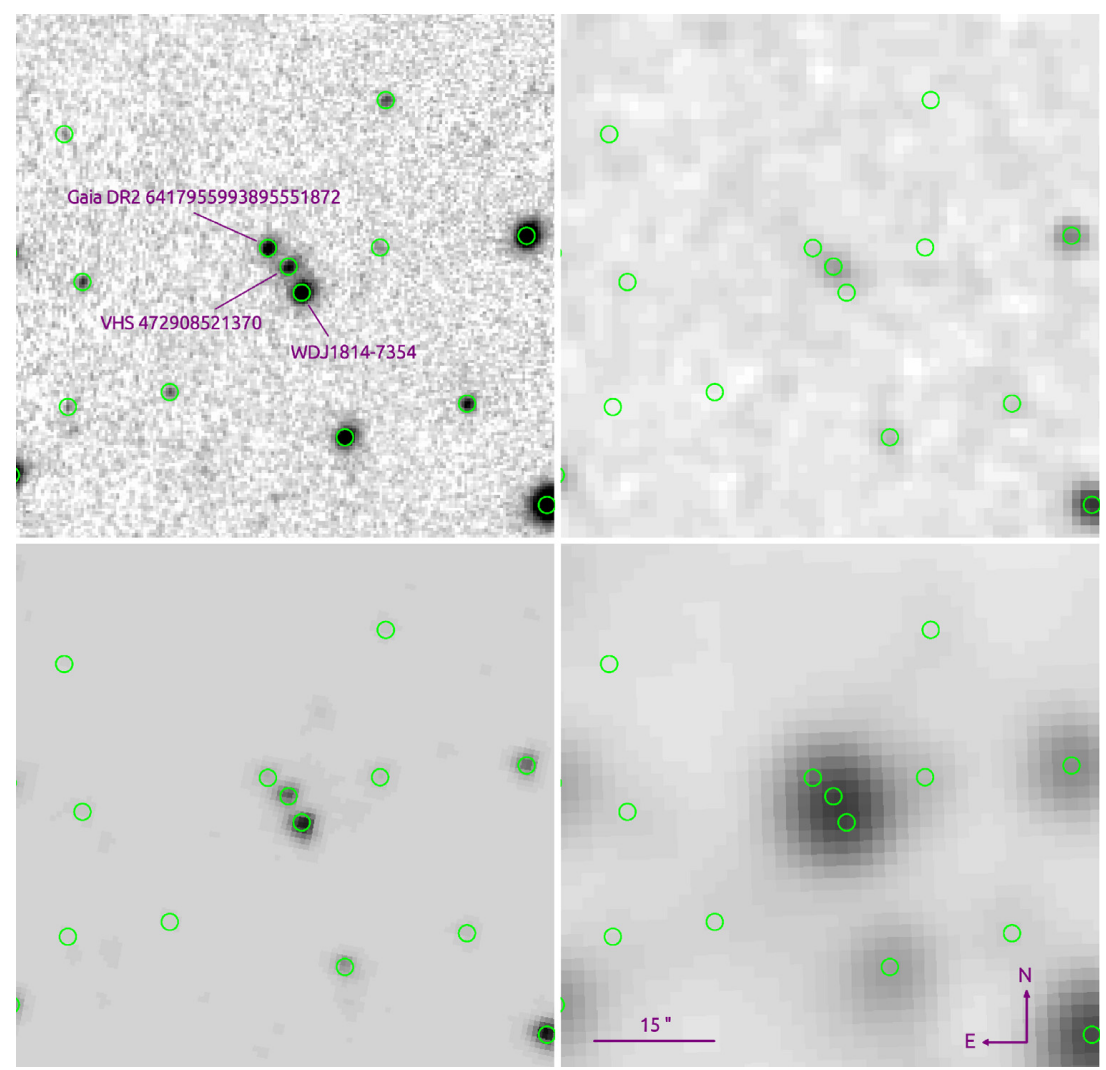

Figure 2. Finding charts in different bands centred on the object WDJ1814-7354. From top-left to bottom-right, we display the VHS J-band image, 2MASS J-band image, Spitzer IRAC [3.6]-band image, and WISE W1-band image. The detected VHS sources are overplotted as green circles, and the orientation and scale are the same in all images. The three sources are labeled in the VHS J image.

Table 3. Gaia DR2 astrometry and photometry of the object Gaia DR2 6417955993895551872, along with its distance (d) in parsecs from Bailer-Jones et al. (2018). Barycentric right ascension $(\alpha)$ and declination $(\delta)$ are in the International Celestial Reference System and at Gaia DR2 reference epoch 2015.5. Proper motion in right ascension $\left(\mu_{\alpha} \cos \delta\right)$ and declination $\left(\mu_{\delta}\right)$ and parallax $(\varpi)$ are also at Gaia epoch, 2015.5. Apparent magnitudes are in the three Gaia DR2 passbands.

\begin{tabular}{lc}
\hline Gaia source ID & Gaia DR2 6417955993895551872 \\
\hline$\alpha$ [deg] & 273.57335015736 \\
$\delta$ [deg] & -73.91602610506 \\
$\mu_{\alpha} \cos \delta$ [mas yr $\left.^{-} 1\right]$ & $-7.4 \pm 0.1$ \\
$\mu_{\delta}\left[\mathrm{mas} \mathrm{yr}{ }^{-} 1\right]$ & $4 \pm 1$ \\
$\varpi[\mathrm{mas}]$ & $-1.8 \pm 0.8$ \\
$\mathrm{G}[\mathrm{mag}]$ & $19.877 \pm 0.008$ \\
$\mathrm{G}_{\mathrm{BP}}[\mathrm{mag}]$ & $20.62 \pm 0.08$ \\
$\mathrm{G}_{\mathrm{RP}}[\mathrm{mag}]$ & $18.67 \pm 0.03$ \\
$\mathrm{G}_{\mathrm{BP}}-\mathrm{G}_{\mathrm{RP}}[\mathrm{mag}]$ & $1.95 \pm 0.11$ \\
$\mathrm{~d}[\mathrm{pc}]$ & $3200_{-1800}^{+5000}$ \\
\hline
\end{tabular}

as this correction applies to blue sources, being null for red sources like our target.

Apparent magnitudes were computed using Vega zero flux values determined with the Python Pyphot package, that agree with the zero fluxes listed in the IRAC Instrument Handbook $(280.9 \pm 4.1 \mathrm{Jy}$ for IRAC [3.6] and $179.7 \pm 2.6 \mathrm{Jy}$ for IRAC45). The results are presented in Table 5.
Table 4. VHS and unWISE apparent magnitudes and colours of the two other sources identified around the position of WDJ1814-7354.

\begin{tabular}{lcc}
\hline Band (survey) & VHS 472908521370 & Gaia DR2 6417955993895551872 \\
\hline $\mathrm{J}$ (VHS) [mag] & $17.78 \pm 0.04$ & $17.263 \pm 0.025$ \\
Ks (VHS) [mag] & $16.18 \pm 0.04$ & $16.52 \pm 0.06$ \\
$\mathrm{~J}-$ Ks [mag] & $1.6 \pm 0.08$ & $0.74 \pm 0.08$ \\
W1 (unWISE) & $15.144 \pm 0.011$ & - \\
[mag] & & - \\
W2 (unWISE) & $14.643 \pm 0.024$ & - \\
[mag] & & - \\
W1 - W2 [mag] & $0.50 \pm 0.03$ & \\
\hline
\end{tabular}

Table 5. Spitzer extracted photometry for WDJ1814-7354 and VHS 472908521370. $f_{36}$ and $f_{45}$ are the fluxes in Spitzer IRAC [3.6] and IRAC [4.5] bands in $\mu \mathrm{Jy}$ units obtained using MOPEX and $m_{36}$ and $m_{45}$ are the apparent magnitudes computed from these fluxes.

\begin{tabular}{lcc}
\hline & WDJ1814-7354 & VHS 472908521370 \\
\hline$f_{36}[\mu \mathrm{Jy}]$ & $591 \pm 7$ & $297 \pm 5$ \\
$f_{45}[\mu \mathrm{Jy}]$ & $643 \pm 9$ & $353.2 \pm 6$ \\
$m_{36}[\mathrm{mag}]$ & $14.185 \pm 0.013$ & $14.933 \pm 0.017$ \\
$m_{45}[\mathrm{mag}]$ & $13.622 \pm 0.015$ & $14.272 \pm 0.017$ \\
\hline
\end{tabular}

\subsection{FIRE spectroscopy}

We explored the possibility of VHS 472908521370 being a cool companion of WDJ1814-7354. If we place it at the same distance 


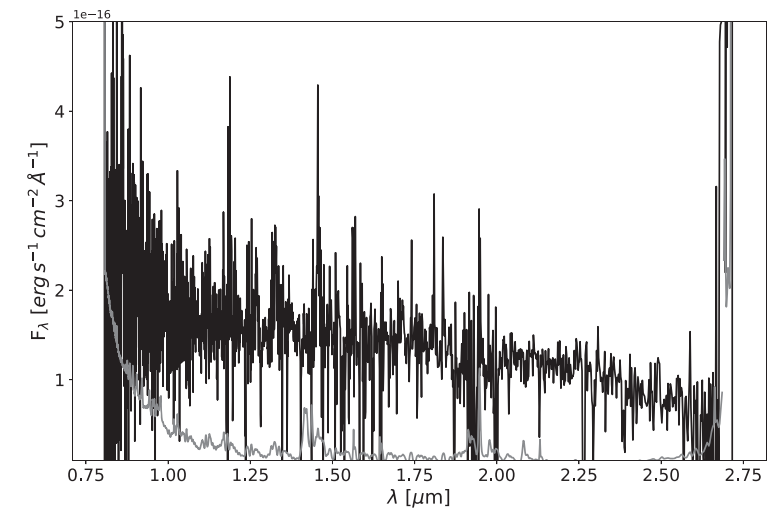

Figure 3. Spectrum of VHS 472908521370 obtained on the night of 2019 June 2 with the FIRE instrument. In black, the combination of the individual spectra using a robust weighted mean, and in grey, the formal error in flux were shown.

as WDJ1814-7354, its absolute magnitudes in J and Ks VHS bands and in Spitzer bands would be consistent with a brown dwarf of spectral type L5.5-L6, according to the relations from Dupuy \& Liu (2012; Table 14). To discard or confirm this possibility, we obtained a spectrum of this object with the Folded-port InfraRed Echellette (FIRE, Simcoe et al. 2013) at the Magellan Baade telescope, Las Campanas observatory, on the night of 2019 June 2. We used the low-resolution prism mode, with a 0.6 " wide slit, to obtain eight exposures with a total integration time of 1732 seconds (28.8 min). We used the typical ABBA nodding pattern.

We used the FIREHOSE pipeline (Fica et al. 2016) to reduce the spectra which include: flat fielding, wavelength calibration, A-B pair subtraction to remove first-order sky emission. We then trace the spectra in each positive and negative feature and remove the residual background emission using the flux that fell on the slit. We then combined the eight individual spectra using a robust weighted mean. For telluric correction and flux calibration, we used the A0V star HD 167061 and we reduced following an identical procedure than for the object and to construct and apply the telluric correction we used the IDL-based task XTELLCOR (Vacca, Cushing \& Rayner 2003).

The FIRE spectrum is shown in Fig. 3. Although the SNR is $\sim 1.9$, it does not show any evident characteristic features of substellar objects, such as strong water bands, iron hydrates $(\mathrm{FeH}), \mathrm{NaI}, \mathrm{CO}$, or methane absorption. We therefore conclude that VHS 472908521370 is likely another background object. It is brighter in the IR and closer to the white dwarf than Gaia DR2 6417955993895551872, and it is likely contributing to $2 \mathrm{MASS}$ and WISE photometry.

\section{ANALYSIS}

\subsection{White dwarf properties}

The spectrum of WDJ1814-7354 is that of a typical DAZ white dwarf, characterized by broad Balmer lines and absorption from $\mathrm{Mg}$ I-II and Ca I-II species and weaker absorption lines from Fe I. Examples of $\mathrm{Mg}$ and $\mathrm{Ca}$ lines in both 2011 and 2019 spectra are shown in Figs 4-5. Fe lines are not shown in the figures due to their faintness, but with a large number of them and consistent results we are confident of the Fe detection.

We analysed the combined UVB and VIS 2011 spectra via comparison with a grid of synthetic spectra modelled with the stellar atmosphere code outlined by Koester (2010), Koester, Kepler \& Irwin (2020). The NIR spectrum was not used due to its low SNR. The
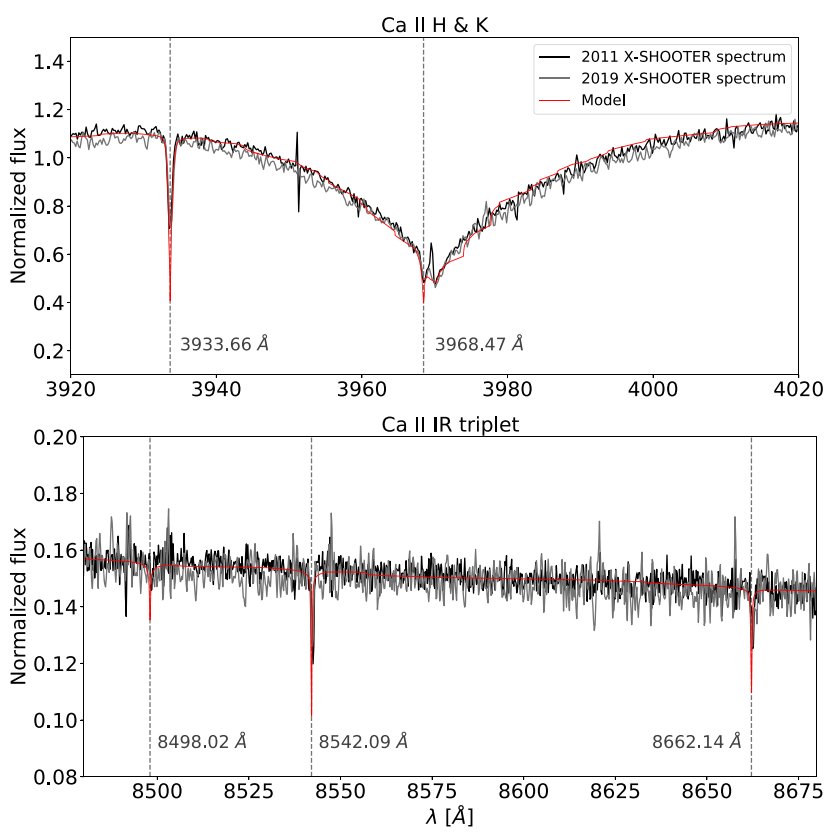

Figure 4. Calcium absorption lines found in WDJ1814-7354 X-SHOOTER 2011 (black) and 2019 (grey) spectra, marked with grey vertical dashed lines. The white dwarf model is superimposed in red. Line wavelength values from NIST Atomic Spectra Database (Kramida et al. 2019).
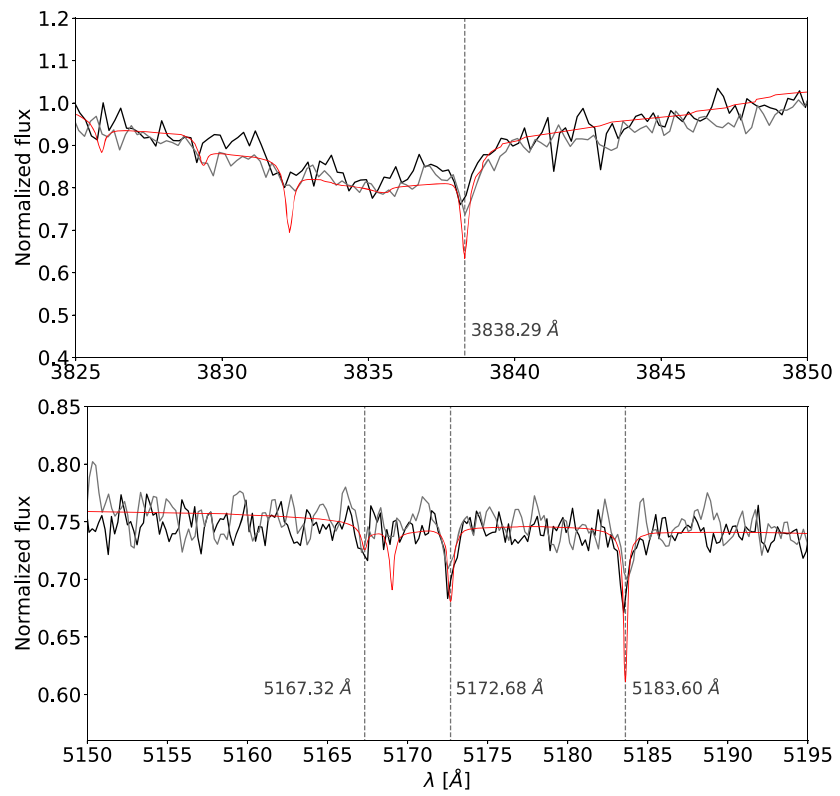

Figure 5. Mg I absorption lines found in WDJ1814-7354 X-SHOOTER 2011 (black) and 2011 (grey) spectra, marked with grey vertical dashed lines. The white dwarf model is superimposed in red. Line wavelength values from NIST Atomic Spectra Database (Kramida et al. 2019).

2019 spectrum was used to investigate the potential variability of metal absorption lines within the 1-sigma errors (see Section 4.2).

We started by fitting the full Balmer series, incrementally including the identified pollutants after finding the initial best fit and subsequently adjusting the result. The best-fit is obtained for $T_{\text {eff }}=10$, $140 \mathrm{~K}$, and $\log g=8.10$. Statistical uncertainties are negligible due to the high SNR of the optical spectrum. Subsequently, we performed 
Table 6. Radial velocities computed in both 2011 and 2019 XSHOOTER spectra. The average values are $\overline{\mathrm{RV}}_{2011}=46 \pm 8 \mathrm{~km}$ $\mathrm{s}^{-1}$ and $\overline{\mathrm{RV}}_{2019}=45 \pm 10 \mathrm{~km} \mathrm{~s}^{-1}$.

\begin{tabular}{lcc}
\hline Line (wavelength) $[\AA]$ & $\mathrm{RV}_{2011}\left[\mathrm{~km} \mathrm{~s}^{-1}\right]$ & $\mathrm{RV}_{2019}\left[\mathrm{~km} \mathrm{~s}^{-1}\right]$ \\
\hline Ca II (3933.66) & 46 & 54 \\
Ca II (3968.47) & 58 & 54 \\
Ca II (8662.14) & 47 & 31 \\
Ca I (4226.73) & 39 & 48 \\
Mg I (3838.29) & 41 & 52 \\
Mg I (5167.32) & 42 & 41 \\
Mg I (5172.68) & 46 & 40 \\
Mg I (5183.69) & 43 & 56 \\
\hline
\end{tabular}

a photometric fit of the available APASS and Gaia DR2 photometry (Table 2), in which we used the Gaia DR2 parallax and the white dwarf mass-radius relation (Fontaine, Brassard \& Bergeron 2001) as additional priors. We have not used VHS photometry to be sure that the IR excess has no effect in the fit. The photometric fit delivers a 0.1 dex-lower surface gravity. Hence, we iterated between spectroscopic ( $\log g$ fixed) and photometric ( $T_{\text {eff }}$ fixed) fits, until both methods converged to a final solution. The difference between the initial spectroscopic fit and the final result is adopted as the error estimate, resulting in $T_{\text {eff }}=10,090 \pm 50 \mathrm{~K}$ and $\log g=8.00 \pm 0.13$. The interstellar reddening obtained as a result of our fitting procedure is negligible, and of the order of $0.01 \mathrm{mag}$.

We measured the radial velocities of the strongest metal lines via fitting Gaussian models and estimated the uncertainty as the scatter between the measurements of the different lines. For the 2011 spectrum, we obtained an average radial velocity of $\overline{\mathrm{RV}}_{2011}=$ $44 \pm 5 \mathrm{~km} \mathrm{~s}^{-1}$ and for the 2019 spectrum, $\overline{\mathrm{RV}}_{2019}=47 \pm 8 \mathrm{~km} \mathrm{~s}^{-1}$ The individual radial velocities for the different lines are presented in table 6 .

The atmospheric parameters of WDJ1814-7354 correspond to a white dwarf mass of $0.59_{-0.06}^{+0.08} \mathrm{M}_{\odot}$ and a cooling age of $\tau_{\text {cool }}=$ $0.58_{-0.07}^{+0.14}$ Gyr. Using the Cummings et al. (2018) initial-to-finalmass relation (IFMR), we infer a progenitor mass of $1.3_{-0.4}^{+0.5} \mathrm{M}_{\odot}$. To estimate the progenitor age, we used the evolutionary tracks of Choi et al. (2016). For a progenitor mass of $1.3 \mathrm{M}_{\odot}$ (late F-type star), the progenitor age is $\tau_{\mathrm{MS}}=5.2 \mathrm{Gyr}$, and the total age of the white dwarf would be $\tau_{\mathrm{MS}}+\tau_{\text {cool }}=5.8 \mathrm{Gyr}$. The large systematic uncertainty implied by the empirical IFMR translates into a large uncertainty on the progenitor age. For the upper limit of the progenitor mass of $1.8 \mathrm{M}_{\odot}$ (a late A-type star), the progenitor age would be $\tau_{\mathrm{MS}}=$ $3.4 \mathrm{Gyr}$, so the lower limit on the total age of the white dwarf is $4 \mathrm{Gyr}$. The upper limit on the total age is unconstrained, because the lower limit of the progenitor mass is $0.9 \mathrm{M}_{\odot}$ (an early $\mathrm{K}$ dwarf star), whose lifetime could be comparable or longer than the age of the Universe.

\subsection{Composition of the accreted material and diffusion time-scales}

The abundances of the three detected elements $(\mathrm{Mg}, \mathrm{Ca}$, and $\mathrm{Fe})$ are given in Table 7. The listed abundances and uncertainties are the averages and the standard deviations inferred from the modelling of the strongest lines for each detected element: lines at 3933.66, 3968.47, 8498.02, 8542.09, 8662.14 (Ca II), and 4226.73 $\AA$ (Ca I) for Calcium; lines at 4481.13 (Mg II), 3838.29, 5167.32, 5172.68, and $5183.69 \AA$ (Mg I) for Magnesium; and lines at 3609, 3619, 3632, $3720,3738,3750,3759,3816,3821$, and $3826 \AA$ for iron (Fe I). In addition, we derive upper limits for $\mathrm{Na}, \mathrm{Al}, \mathrm{Si}, \mathrm{P}, \mathrm{S}, \mathrm{Ti}$, and $\mathrm{Ni}$.
Table 7. Metal abundances measured in WDJ1814-7354 XSHOOTER spectrum from 2011. $[\mathrm{Z} / \mathrm{H}]=\log n(\mathrm{Z}) / \mathrm{n}(\mathrm{H})$ is the abundance of the element $\mathrm{Z}, \tau_{d}$ is the diffusion time-scale and $\dot{M}$ is the accretion flux. The total accretion flux includes only the observed elements, not the upper limits.

\begin{tabular}{lclc}
\hline Element & {$[\mathrm{Z} / \mathrm{H}]$} & $\tau_{d}[\mathrm{yrs}]$ & $\log |\dot{\mathrm{M}}|\left[\mathrm{g} \mathrm{s}^{-1}\right]$ \\
\hline $\mathrm{Na}$ & $<-7.40$ & 22.42 & $<7.124$ \\
$\mathrm{Mg}$ & $-6.14 \pm 0.08$ & 21.66 & $8.42 \pm 0.08$ \\
$\mathrm{Al}$ & $<-7.30$ & 19.78 & $<7.35$ \\
$\mathrm{Si}$ & $<-6.00$ & 19.32 & $<8.67$ \\
$\mathrm{P}$ & $<-4.50$ & 17.701 & $<10.26$ \\
$\mathrm{~S}$ & $<-4.50$ & 17.33 & $<10.28$ \\
$\mathrm{Ca}$ & $-7.22 \pm 0.15$ & 14.415 & $7.74 \pm 0.15$ \\
$\mathrm{Ti}$ & $<-8.00$ & 12.21 & $<7.12$ \\
$\mathrm{Fe}$ & $-6.06 \pm 0.19$ & 10.814 & $9.17 \pm 0.19$ \\
$\mathrm{Ni}$ & $<-6.30$ & 10.44 & $<8.96$ \\
Total & - & - & $9.25 \pm 0.17$ \\
\hline & & &
\end{tabular}

Using the accretion-diffusion models of Koester (2009), we estimate a mass-fraction contained within the convection zone of $\log M_{\mathrm{cvz}} / M_{\mathrm{wd}}=-11.1$. The diffusion time-scales of the three detected pollutants are of the order of 10-20 yr. Given these relatively short diffusion time-scales, we tested whether any measurable changes occurred during the $\approx 10$-yr baseline between our first observation and the more recent 2019 spectrum. For the three strongest lines of $\mathrm{CaII}$ and $\mathrm{MgI}$, there is an indication that the equivalent widths of 2019 spectrum are smaller by 3-6 per cent than in 2011. The lines have the same widths, but are slightly less deep, which might be caused by a small difference in resolution of the two spectra. This difference is marginally compatible with the mutual errors. For the smaller lines, any differences are within the errors. If the differences are real, they would correspond to differences in the abundances by about $0.02-0.04$ dex, within the errors of our determinations. We interpret this result as an indication of WDJ1814-7354 currently accreting from its debris disc.

In Table 7, we list the diffusion time-scales, defined as the time necessary for the flux to be reduced by $1 / \mathrm{e}$, corresponding to the model atmosphere of WDJ1814-7354 and the accretion fluxes we measure for each detected elements as well as those with upper limits. The accretion flux is defined as $\dot{\mathrm{M}}=M_{\mathrm{wd}} \times 10^{q} \times A \times 10^{[\mathrm{Z} / \mathrm{H}]} / \tau_{d}$, where $M_{\mathrm{wd}}$ is the mass of the white dwarf, $A$ is the atomic weight, and $q=\log _{10}\left(M_{\mathrm{CVZ}} / M_{\mathrm{wd}}\right)$, being $M_{\mathrm{CVZ}}$ the mass of the convection zone. The systematic errors from the parameters on the accretion fluxes largely cancel when we use abundance ratios.

\subsection{Disc model fit}

The IR excess of WDJ1814-7354 detected by 2MASS and WISE persists in the higher-resolution photometry of VHS, unWISE, and Spitzer (see Fig. 6).

We have estimated the IR excess as the difference between a blackbody fit to the white dwarf model scaled to Gaia band $\mathrm{G}_{\mathrm{RP}}$ photometry and the resolved photometry (VHS, unWISE, and Spitzer). We have modelled this IR excess with a simple blackbody function:

$B_{\lambda}=\alpha \frac{2 \pi c^{2}}{\lambda^{5}} \frac{1}{e^{\frac{h c}{\mathrm{~K}_{\mathrm{B}} \mathrm{bb}^{\lambda}}}-1}$

where $h$ is the Plank constant, $c$ the speed of light, $\mathrm{K}_{\mathrm{B}}$ the Boltzmann constant, $\mathrm{T} \_b b$ the temperature of the blackbody, and $\alpha$ is the scaling factor. 


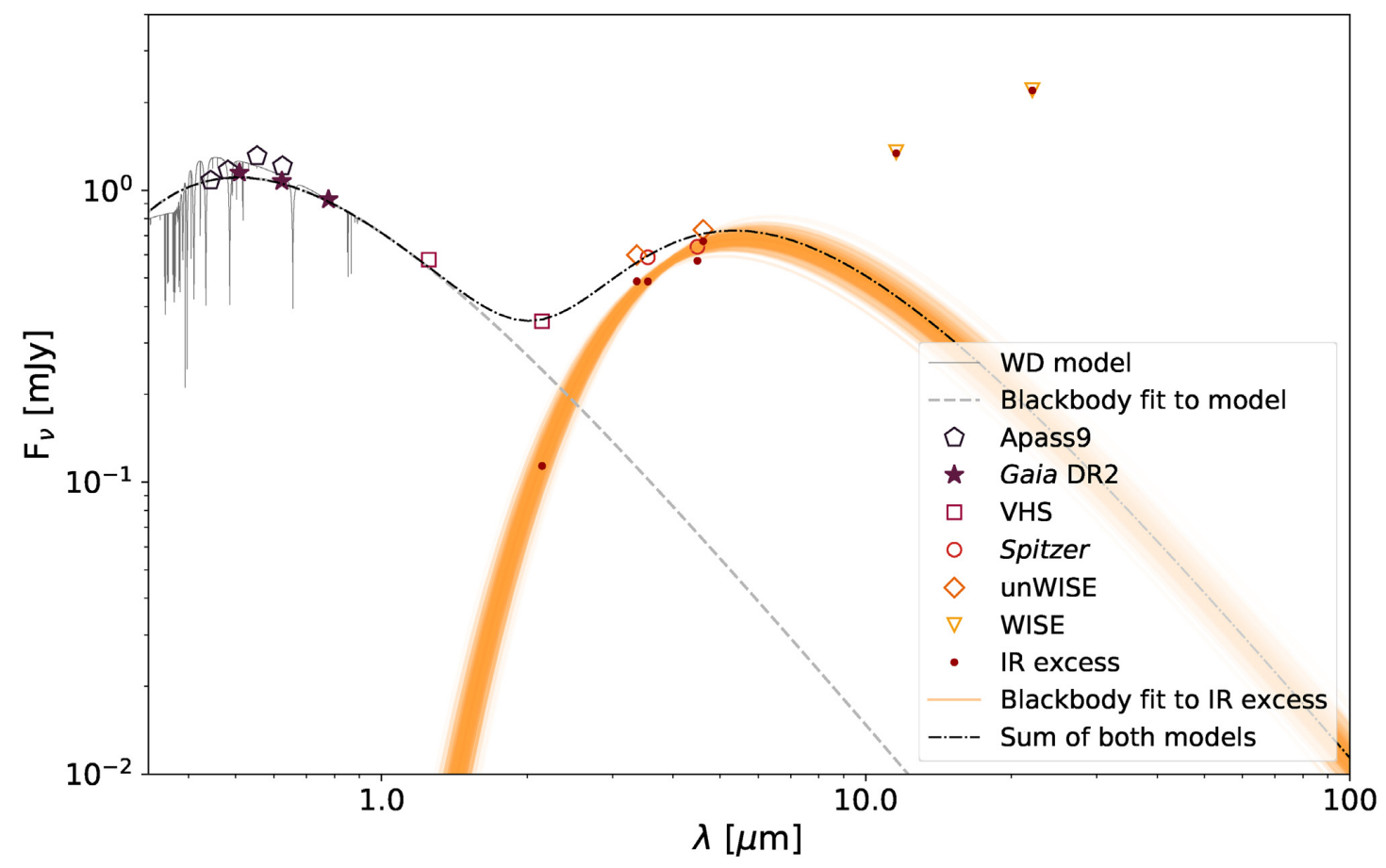

Figure 6. WDJ1814-7354 spectral energy distribution and IR excess along with a random set of 500 blackbody fits to the IR excess and the sum of the disc model (blackbody fit to the median of the parameter distribution) and the white dwarf backbody model (dashed-dotted line) expressed in flux density units (mJy). The WISE fluxes are upper limits, as WDJ1814-7354 photometry is blended with the background sources in WISE bands.

We have used a blackbody model instead of a Flat Ring Model because we lack reliable photometry for wavelengths longer than 4.5 $\mu \mathrm{m}$, necessary to properly constrain the inner and outer temperatures of the disc. We note the limitations of using a single temperature blackbody to model the dust and that more detailed modelling is required to fully constrain the properties of the disc.

For computing the parameters of the disc blackbody model fit (temperature $\mathrm{T}_{\mathrm{bb}}$ of the disc and scaling factor $\alpha$ ) and their uncertainties, we have produced a set of 10000 simulated random data points drawn from a Gaussian distribution, with mean $\mu$ equal to each IR excess value (excluding the blended 2MASS and WISE photometry) and $\sigma$ equal to its corresponding error, obtained after a linear propagation of the errors from the photometry and the previous blackbody fit to the white dwarf model.

We have fit the blackbody model to each of the 10000 datasets and we have obtained the two parameter distributions shown in Fig. 7. The effective temperature distribution is a Gaussian distribution, whose median and standard deviation is $T_{\text {eff }}=910 \pm 50 \mathrm{~K}$. The scaling factor is proportional to $\mathrm{R}_{\mathrm{bb}} / \mathrm{d}$, where $\mathrm{R}_{\mathrm{bb}}$ is the radius of the blackbody and $\mathrm{d}$ the distance to the system. The mean value of the distribution of the scaling gives a rough estimate of $\mathrm{R}_{\mathrm{bb}} \sim 10^{11} \mathrm{~cm} \sim 100 \mathrm{R} \oplus$.

In Fig. 6, a random set of 500 blackbody fits is shown along with the IR excess data points, the photometry, the scaled white dwarf model and its blackbody fit and the sum of this fit and the disc model corresponding to the obtained parameters.. We can see that the resolved photometry is reasonably well fitted with this model. The reduced $\chi^{2}$ of the fit to the IR excess is $\sim 2.5$.

\section{DISCUSSION}

The discovery of the disc around WDJ1814-7354 was serendipitous, as the initial search in which this object was found was aiming to identify white dwarf + ultracool dwarf pairs. This
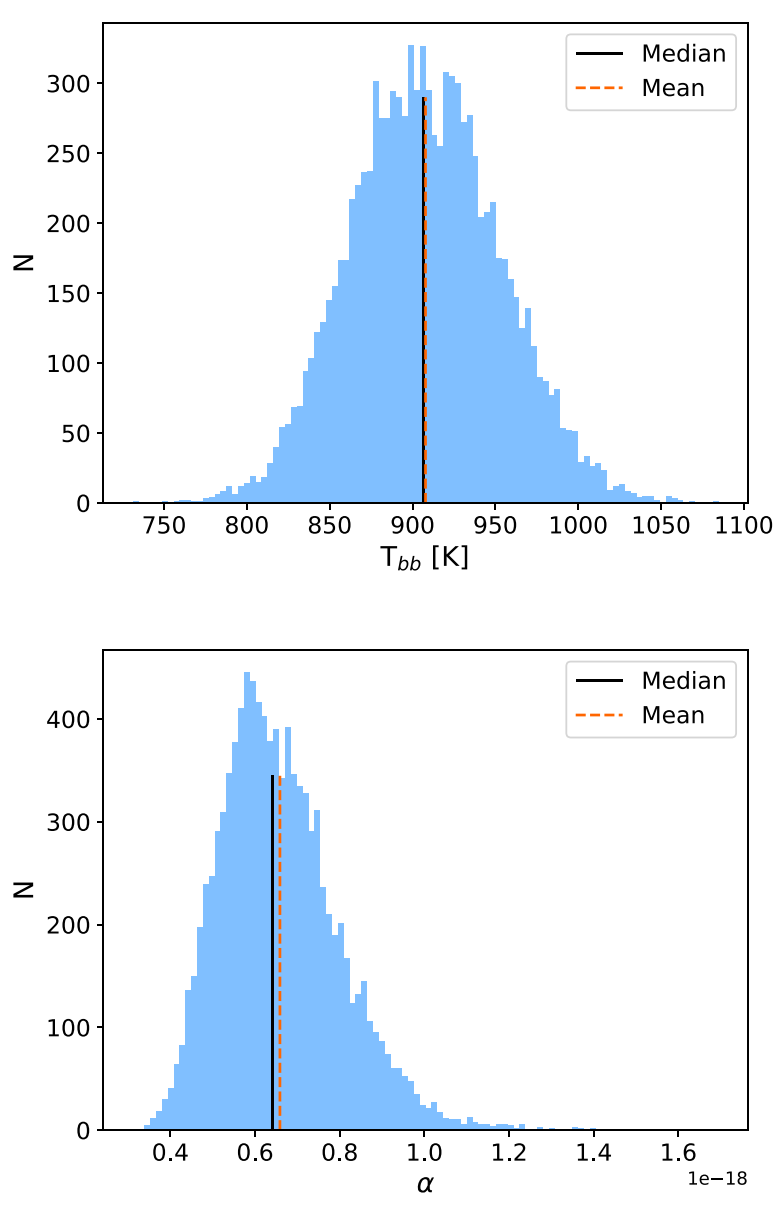

Figure 7. Distribution of obtained parameters from the blackbody fit to the 10000 simulated datasets. 


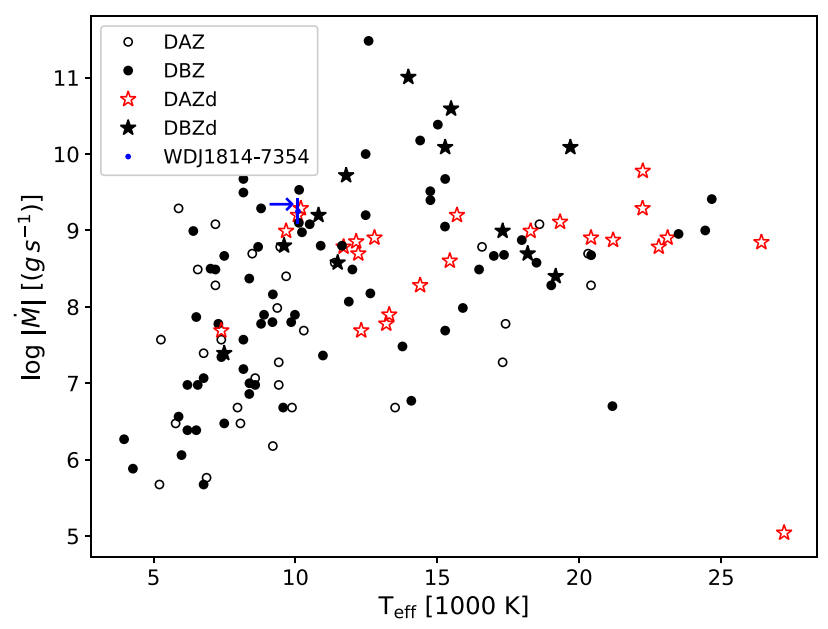

Figure 8. Time-averaged dust accretion rates versus effective temperature of literature white dwarfs with the value for WDJ1814-7354 over-plotted with a blue dot symbol, and a blue arrow pointing at its location for visibility. DAZ- and DBZ-type stars are plotted as open and filled circles, respectively, while objects with IR excess are displayed as open and filled stars rather than circles. This figure is based on Fig. 10 from Farihi (2016), updated with data from Swan et al. (2019b) and Xu et al. (2019).

object was not found in previous searches for discs around white dwarfs. An explanation could be that the background objects made its IR excess unlikely to be due to a disc, until the photometry was recently deblended. This is an issue for WISE selected white dwarfs with IR excesses, as noted recently by Dennihy et al. (2020b).

The total estimated accretion flux of $\dot{M}=(1.8 \pm 0.7) \times 10^{9} \mathrm{~g} \mathrm{~s}^{-1}$, presented in Table 7, is higher than the average value for DAZ-type objects with detected discs, (see table 6 of Xu et al. 2019), as can be also seen in Fig. 8. In this figure, we have placed WDJ1814-7354 in Fig. 10 of Farihi (2016), updated with data from Swan et al. (2019b) and $\mathrm{Xu}$ et al. (2019), to compare it with other white dwarfs with measured accretion rates from the literature. It has the fourth highest accretion flux of the DAZd type (defined by von Hippel et al. 2007 as white dwarf of DAZ type with a detected circumstellar disc), and this flux is higher than 83.8 per cent of all the other white dwarfs shown in the plot. We can see that WDJ1814-7354 is also one of the coolest (hence, with longer cooling ages) DAZ white dwarfs with discs known to date, with only 16 per cent of this sample having lower temperatures, and it is the third coolest of the known sample of 17 DAZd white dwarfs.

We have estimated the fractional disc luminosity $\left(\tau=L_{\mathrm{IR}} / L_{*}\right)$ of our system by calculating the bolometric fluxes of the IR excess and the white dwarf. To do so, we have integrated the blackbody models of the IR excess and the white dwarf within the limits where the flux drops 99.99 per cent. The uncertainty for each bolometric flux has been estimated as the standard deviation of a distribution of 10000 blackbody models created by randomly varying the model parameters within their errors. We have compared the fractional luminosity obtained with that of other systems from the literature in Fig. 9. We can see that WDJ1814-7354 disc luminosity fraction is among the largest of the discovered systems. In this plot it is the largest for cool white dwarfs (with $T_{\text {eff }}<15000 \mathrm{~K}$ ), although many systems from the literature do not have fractional luminosities available and were not included in this plot, and the available data

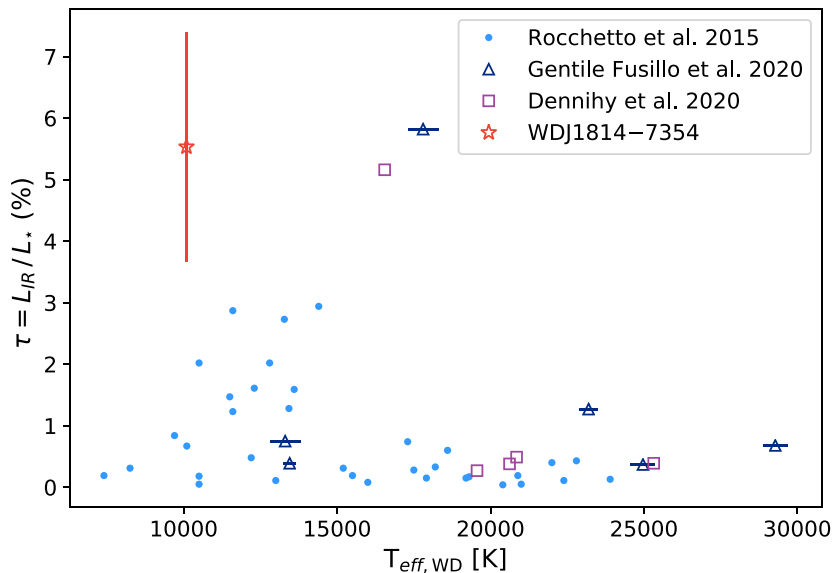

Figure 9. Percentages of fractional disc luminosity versus effective temperature of the central white dwarf for our system compared with data from Rocchetto et al. (2015), Gentile Fusillo et al. (2020a), and Dennihy et al. (2020a).

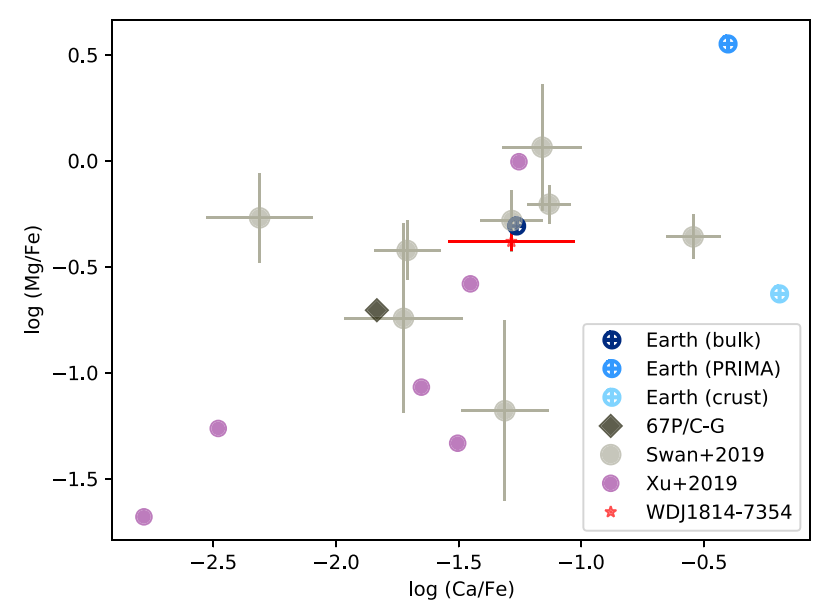

Figure 10. Comparison between the log mass abundance ratio of $\mathrm{Mg}$ and $\mathrm{Ca}$ for WDJ1814-7354 with white dwarfs and Solar System objects, from Swan et al. (2019a) and Xu et al. (2019). The log mass abundance ratio is defined as $\log _{10} N(\mathrm{X}) / N(\mathrm{Fe})$, obtained from equation (2). Earth abundances are from the bulk silicate Earth (the primitive mantle, representing the composition of the upper layers of the Earth after the core separated, abbreviated PRIMA in the figure), bulk Earth and Earth crust.

for the rest does not include uncertainties, so the comparison has to be taken with caution.

Finally, under the assumption that WDJ1814-7354 is currently accreting debris from a circumstellar reservoir in a steady state, i.e. with equilibrium between accretion and diffusion, we can estimate the accreted body composition, relative to $\mathrm{Fe}$, via the following equation, (cf. Koester 2009; Gänsicke et al. 2012):

$\frac{N(\mathrm{X})}{N(\mathrm{Fe})}=\frac{\dot{\mathrm{M}}(X) A(\mathrm{Fe})}{\dot{\mathrm{M}}(\mathrm{Fe}) A(\mathrm{X})}$

where $N(\mathrm{X})$ is the number abundance of element $\mathrm{X}$ and $A(\mathrm{X})$ its atomic weight.

Thus, we can compare the composition of the accreted material of WDJ1814-7354 with that of Solar System bodies and of other polluted white dwarfs from the literature (see Fig. 10). A steady state can be assumed to be valid for white dwarf atmospheres that are char- 
acterized by short diffusion time-scales, like for WDJ1814-7354, and systems that do not show line or disc variability. Veras \& Heng (2020), Girven et al. (2012), Farihi et al. (2012) show that disc lifetimes are expected to be much longer than such diffusion timescales. In our case, the disc could have been building up material for $\sim 10$ years, and it is expected not to show a change in its composition if the accretion time-scale is longer than the diffusion time-scale.

The material being accreted by WDJ1814-7354 has similar metal ratios of $\mathrm{Ca} / \mathrm{Fe}$ and $\mathrm{Mg} / \mathrm{Fe}$ like those of bulk Earth, as can be seen in Fig. 10. The upper limits available for other elements do not allow a wider comparison with the chemical profile of known Solar System bodies, as it has been done for other stars in the literature. We cannot accurately determine the total mass of the disc (see e.g Dufour et al. 2010) but we can estimate the mass of the accreted material. By multiplying the order of magnitude of the estimated lifetime of white dwarf discs, $10^{5}$ yrs (Girven et al. 2012), with our measured total accretion rate of detected elements of $\sim 10^{9} \mathrm{~g} \mathrm{~s}^{-1}$, given in Table 7, we obtain $10^{19} \mathrm{~kg}$ of accreted material, which is in the region of masses of Solar System asteroids such as 13 Egeria (Baer, Chesley \& Matson 2011) or 48 Bamberga (Carry 2012). This finding supports the evidence that rocky minor planetary bodies with Earth-like composition form around Sun-like stars elsewhere.

\section{SUMMARY}

We have presented a spectroscopic and photometric analysis of the white dwarf WDJ1814-7354, an object originally identified to have a strong IR excess in the 2MASS and WISE catalogues. We confirmed this IR excess to be intrinsic to the white dwarf, and likely corresponding to the emission of a dusty disc around the star. The finding of $\mathrm{Ca}, \mathrm{Fe}$, and $\mathrm{Mg}$ absorption lines in the X-SHOOTER spectrum of the white dwarf is further evidence of accretion from a dusty disc.

We have compared the circumstellar metal lines in two spectra taken 8 years apart, finding no significant changes in radial velocities or in equivalent widths. This is not surprising, as the diffusion time-scales derived in Section 4.2 for $\mathrm{Mg}, \mathrm{Ca}$, and $\mathrm{Fe}$ go from 10 to 22 years, and as there has not yet been found unambiguously variability in white dwarf absorption metallic lines. Due to the blended photometry for this object in most IR surveys, we cannot study the potential variability of WDJ1814-7354 in the IR without follow-up observations.

The ratio of the disc and white dwarf luminosities is among the highest from the literature for a relatively cool white dwarf. From the analysis of the composition of the accreted material and the estimated total accretion rate, we suggest the possibility that a minor body was tidally disrupted into forming the disc of debris material around WDJ1814-7354.

\section{ACKNOWLEDGEMENTS}

We thank the anonymous referee for a quick and adequately thorough revision of this manuscript. EGE acknowledges Astronomers for Planet Earth ${ }^{3}$ and recent works that raise awareness about $\mathrm{CO}_{2}$ emissions from our professional activities (see e.g Burtscher et al. 2020; Stevens et al. 2020) and commits to not travel anywhere by aeroplane for the purpose of promoting this paper. EGE also thanks Andrew Swan and astronomy $\mathrm{PhD}$ students in the University of Hertfordshire for helpful discussions. We thank Dr. Siyi Xu and

${ }^{3}$ https://astronomersforplanet.earth/
Dr. Amy Bonsor for kindly sharing their recent X-SHOOTER and Spitzer observations of this white dwarf. We thank Beth Klein and Jay Farihi for a helpful discussion that improved this article.

EGE and WJC are supported by a University of Hertfordshire PhD studentship. Part of this work was possible thanks to the support of the Royal Astronomical Society (RAS) via the RAS grant E. A. Milne Travelling Fellowship awarded to EGE. RR has received funding from the postdoctoral fellowship programme Beatriu de Pinós, funded by the Secretary of Universities and Research (Government of Catalonia) and by the Horizon 2020 programme of research and innovation of the European Union under the Maria SkłodowskaCurie grant agreement number 801370. FM acknowledges support from the NASA Postdoctoral Program at the Jet Propulsion Laboratory, administered by Universities Space Research Association under a contract with NASA. FM also acknowledges support from grant \#80NSSC20K0452 under the NASA Astrophysics Data Analysis Program. JCB acknowledges support from FONDECYT postdoctorado 3180716. LKR would like to acknowledge funding from The Science and Technology Facilities Council and the Institute of Astronomy, Cambridge.

Based on observations collected at the European Southern Observatory under ESO programmes 087.C-0639(B) (PI Dr. Avril Day-Jones) and 0103.C-0431(B) (PI Dr. Siyi Xu, Rogers et al. in prep.), and observations by Spitzer space telescope, Director's Discretionary Time (DDT) program ID 14220, with PI Dr. Siyi $\mathrm{Xu}$ (Lai et al. in prep.). This work has made use of data from the European Space Agency (ESA) mission Gaia (https://www.cosmos .esa.int/gaia), processed by the Gaia Data Processing and Analysis Consortium (DPAC, https://www.cosmos.esa.int/web/gaia/dpac/con sortium). Funding for the DPAC has been provided by national institutions, in particular the institutions participating in the Gaia Multilateral Agreement. The VISTA Data Flow System pipeline processing and science archive are described in Irwin et al. (2004), Hambly et al. (2008), and Cross et al. (2012). We have used data from the 6th data release (VHSDR6).

This research made use of NUMPY (Harris et al. 2020), SCIPY (Virtanen et al. 2020), ASTROPY, (Astropy Collaboration et al. 2013), MAT-PLOTLIB, (Hunter 2007), PANDAS (Wes McKinney 2010), and SAOImage DS9 (Smithsonian Astrophysical Observatory 2000).

\section{DATA AVAILABILITY}

All data underlying this article is publicly available from the relevant observatory archive (see Section 3 and acknowledgements) or it is available on https://doi.org/10.5281/zenodo.4268013

\section{REFERENCES}

Aannestad P. A., Sion E. M., 1985, AJ, 90, 1832

Astropy Collaboration, 2013, A\&A, 558, A33

Baer J., Chesley S. R., Matson R. D., 2011, AJ, 141, 143

Bailer-Jones C. A. L., Rybizki J., Fouesneau M., Mantelet G., Andrae R., 2018, AJ, 156, 58

Barber S. D., Kilic M., Brown W. R., Gianninas A., 2014, ApJ, 786, 77

Bruhweiler F. C., Kondo Y., 1981, ApJ, 248, L123

Burtscher L., Barret D., Borkar A. P., Grinberg V., Jahnke K., Kendrew S., Maffey G., McCaughrean M. J., 2020, Nat. Astron., 4, 823

Carry B., 2012, Planet. Space Sci., 73, 98

Choi J., Dotter A., Conroy C., Cantiello M., Paxton B., Johnson B. D., 2016, ApJ, 823, 102

Cross N. J. G. et al., 2012, A\&A, 548, A119

Cummings J. D., Kalirai J. S., Tremblay P. E., Ramirez-Ruiz E., Choi J., 2018, ApJ, 866, 21 
Cutri R. M. et al., 2013, Explanatory Supplement to the AllWISE Data Release Products.

Day-Jones A. C. et al., 2008, MNRAS, 388, 838

Debes J. H., Hoard D. W., Wachter S., Leisawitz D. T., Cohen M., 2011, ApJS, 197, 38

Debes J. H., López-Morales M., 2008, ApJ, 677, L43

Dennihy E., Clemens J. C., Dunlap B. H., Fanale S. M., Fuchs J. T., Hermes J. J., 2018, ApJ, 854, 40

Dennihy E., Farihi J., Fusillo N. P. G., Debes J. H., 2020b, ApJ, 891, 97

Dennihy E. et al., 2020a, Astrophys. J., 905, ID5

Dufour P., Kilic M., Fontaine G., Bergeron P., Lachapelle F. R., Kleinman S. J., Leggett S. K., 2010, ApJ, 719, 803

Dupuis J., Fontaine G., Pelletier C., Wesemael F., 1993, ApJS, 84, 73

Dupuy T. J., Liu M. C., 2012, ApJS, 201, 19

Farihi J., 2016, New A Rev., 71, 9

Farihi J., Barstow M. A., Redfield S., Dufour P., Hambly N. C., 2010, MNRAS, 404, 2123

Farihi J., Gänsicke B. T., Wyatt M. C., Girven J., Pringle J. E., King A. R., 2012, MNRAS, 424, 464

Farihi J., Jura M., Zuckerman B., 2009, ApJ, 694, 805

Fazio G. G. et al., 2004, ApJS, 154, 10

Fica H. D., Lambrides E., Faherty J., Cruz K. L., BDNYC, 2016, in American Astronomical Society Meeting Abstracts, Vol. 227. p. 146

Finkbeiner D. P. et al., 2004, AJ, 128, 2577

Fontaine G., Brassard P., Bergeron P., 2001, PASP, 113, 409

Fontaine G., Michaud G., 1979, ApJ, 231, 826

Gaia Collaboration et al., 2016, A\&A, 595:A1

Gaia Collaboration et al., 2018, A\&A, 616:A1

Gentile Fusillo N. P. et al., 2020a, preprint (arXiv:2010.13807)

Gentile Fusillo N. P., Tremblay P.-E., Bohlin R. C., Deustua S. E., Kalirai J. S., 2020b, MNRAS, 491, 3613

Girven J., Brinkworth C. S., Farihi J., Gänsicke B. T., Hoard D. W., Marsh T. R., Koester D., 2012, ApJ, 749, 154

Gänsicke B. T., Koester D., Farihi J., Girven J., Parsons S. G., Breedt E., 2012, MNRAS, 424, 333

Gänsicke B. T., Marsh T. R., Southworth J., Rebassa-Mansergas A., 2006, Science, 314, 1908

Gänsicke B. T., Schreiber M. R., Toloza O., Fusillo N. P. G., Koester D., Manser C. J., 2019, Nature, 576, 61

Hambly N. C. et al., 2001, MNRAS, 326, 1279

Hambly N. C. et al., 2008, MNRAS, 384, 637

Harris C. R. et al., 2020, Nature, 585, 357

Hollands M. A., Koester D., Alekseev V., Herbert E. L., Gänsicke B. T., 2017, MNRAS, 467, 4970

Hora J. L., Latter W. B., Allen L. E., Marengo M., Deutsch L. K., Pipher J. L., 2004, ApJS, 154, 296

Hunter J. D., 2007, Comput. Sci. Engin., 9, 90

Irwin M. J. et al., 2004, in Quinn P. J., Bridger A., eds, Society of Photo-Optical Instrumentation Engineers (SPIE) Conference Series, Vol. 5493, Optimizing Scientific Return for Astronomy through Information Technologies. p. 411

Jura M., 2003, ApJ, 584, L91

Jura M., Farihi J., Zuckerman B., 2009, AJ, 137, 3191

Jura M., Young E., 2014, Ann. Rev. Earth Planet. Sci., 42, 45

Koester D., 2009, A\&A, 498, 517
Koester D., 2010, Mem. Soc. Astron. Italiana, 81, 921

Koester D., Kepler S. O., Irwin A. W., 2020, A\&A, 635, A103

Koester D., Rollenhagen K., Napiwotzki R., Voss B., Christlieb N., Homeier D., Reimers D., 2005, A\&A, 432, 1025

Kramida A., Ralchenko Y., Reader J., NIST ASD Team, 2019, NIST Atomic Spectra Database (version 5.7.1) [Online]. https://physics.nist.gov/asd

Makovoz D., Marleau F. R., 2005, PASP, 117, 1113

Manser C. J., Gänsicke B. T., Gentile Fusillo N. P., Ashley R., Breedt E., Hollands M., Izquierdo P., Pelisoli I., 2020, MNRAS, 493, 2127

Manser C. J., Gänsicke B. T., Koester D., Marsh T. R., Southworth J., 2016b, MNRAS, 462, 1461

Manser C. J. et al., 2016a, MNRAS, 455, 4467

McMahon et al., 2019, VizieR Online Data Catalog, II/359

Modigliani A. et al., 2010, Observatory Operations: Strategies, Processes, and Systems III, 7737. p. 773728

Paquette C., Pelletier C., Fontaine G., Michaud G., 1986, ApJS, 61, 197

Probst R. G., 1983, ApJS, 53, 335

Reach W. T. et al., 2005, PASP, 117, 978

Rocchetto M., Farihi J., Gänsicke B. T., Bergfors C., 2015, MNRAS, 449, 574

Rogers L. K., Xu S., Bonsor A., Hodgkin S., Su K. Y. L., von Hippel T., Jura M., 2020, MNRAS, 494, 2861

Schlafly E. F., Meisner A. M., Green G. M., 2019, ApJS, 240, 30

Simcoe R. A. et al., 2013, PASP, 125, 270

Skrutskie M. F. et al., 2006, AJ, 131, 1163

Smithsonian Astrophysical Observatory, 2000, SAOImage DS9: A utility for displaying astronomical images in the $\mathrm{X} 11$ window environment. preprint (ascl:0003.002)

Stevens A. R. H., Bellstedt S., Elahi P. J., Murphy M. T., 2020, Nat. Astron., 4,843

Swan A., Farihi J., Wilson T. G., 2019a, MNRAS, 484, L109

Swan A., Farihi J., Koester D., Holland s M., Parsons S., Cauley P. W., Redfield S., Gänsicke B. T., 2019b, MNRAS, 490, 202

Swan A., Farihi J., Wilson T. G., Parsons S. G., 2020, MNRAS, 496, 5233

Vacca W. D., Cushing M. C., Rayner J. T., 2003, PASP, 115, 389

Veras D., Heng K., 2020, MNRAS, 496, 2292

Vernet J. et al., 2011, A\&A, 536, A105

Virtanen P. et al., 2020, Nat. Meth., 17, 261

Visscher C., Fegley, Bruce J., 2013, ApJ, 767, L12

von Hippel T., Kuchner M. J., Kilic M., Mullally F., Reach W. T., 2007, ApJ, 662,544

Weiler M., 2018, A\&A, 617, A138

Wes McKinney, 2010, in van der Walt S., Millman J., eds, Proc. 9th Python in Sci. Conf.. p. 56

Wright E. L. et al., 2010, AJ, 140, 1868

Wyatt M. C., Farihi J., Pringle J. E., Bonsor A., 2014, MNRAS, 439, 3371

Xu S., Dufour P., Klein B., Melis C., Monson N. N., Zuckerman B., Young E. D., Jura M. A., 2019, AJ, 158, 242

Xu S., Jura M., 2014, ApJ, 792, L39

Xu S., Lai S., Dennihy E., 2020, Astrophys. J., 902, 27

Zuckerman B., Koester D., Reid I. N., Hunsch M., 2003, ApJ, 596, 477

Zuckerman B., Melis C., Klein B., Koester D., Jura M., 2010, ApJ, 722, 725

This paper has been typeset from a $\mathrm{T}_{\mathrm{E}} \mathrm{X} / \mathrm{L} \mathrm{T} \mathrm{T}_{\mathrm{E}} \mathrm{X}$ file prepared by the author. 